\title{
ON DIFFERENTIABLE IMBEDDINGS OF SIMPLY-CONNECTED MANIFOLDS
}

\author{
BY J. LEVINE
}

Communicated by W. S. Massey, June 12, 1963

1. Introduction. We will be concerned with the problem of imbedding (differentiably) a closed simply-connected $n$-manifold $M$ in the $m$-sphere $S^{m}$. According to [3], this problem depends only upon the homotopy type of $M$, in a "stable" range of dimensions. We obtain an explicit equivalent homotopy problem.

We also consider the problem of determining whether two imbeddings of $M$ in $S^{m}$ are isotopic (see [3] for basic definitions). A "homotopy condition" for deciding this question will also be obtained, again in a "stable" range of dimensions.

All manifolds, imbeddings and isotopies are to be differentiable. If $M, V$ are manifolds with boundary and $f$ is an imbedding of $M$ in $V$, it will always be understood that $f(M) \cap \partial V=f(\partial M)$ and the intersection is transverse.

2. Imbedding theorem. $M$ will, hereafter, denote a closed simplyconnected $n$-manifold, $n>4$. Suppose $f$ imbeds $M$ in $S^{m}$; then we can define the normal plane bundle $\nu_{f}$ and, by a construction of Thom [10], an element $\alpha_{f} \in \pi_{m}\left(T\left(\nu_{f}\right)\right)$, where $T\left(\nu_{f}\right)$ is the Thom space (see [10]) of $\nu_{f}$. We call the pair $\left(\nu_{f}, \alpha_{f}\right)$ the normal invariants of $f$. The existence of an imbedding, in particular, implies the existence of an $(m-n)$-plane bundle $\xi$ whose Thom space is reducible in the sense of [1]. It follows from [1] that this property of $M$ is a homotopy invariant and such a bundle $\xi$ must be, a priori, stably fiber homotopy equivalent to the stable normal bundle of $M$.

Let $M_{0}$ denote the complement of an open disk in $M$.

TheOREM 1. Suppose $2 m \geqq 3(n+1)$ and $\xi$ is an $(m-n)$-plane bundle over $M$ stably equivalent to the stable normal bundle of $M$, such that $T(\xi)$ is reducible. Then there is an imbedding $f$ of $M$ in $S^{m}$ such that $\nu_{f}$ is fiber homotopy equivalent to $\xi$ :

(a) Over $M$ if $n=6,14$ or $n \neq \equiv 2 \bmod 4$.

(b) Over $M_{0}$ if $n \equiv 2 \bmod 4$.

It is to be expected that the conclusion of (a) is valid for all $n$. The difficulty in the proof arises from the lack of a satisfactory general definition of the Arf invariant (see [7]). In certain special cases, e.g., if $M$ is a $\pi$-manifold or $\pi_{i}(M)=0$ for $2 i<n$, we can obtain the conclusion of (a). 
3. Isotopy theorems. Suppose $f, g$ are isotopic imbeddings of $M$ in $S^{m}$. It is easy to show that there is a bundle map $\phi: \nu_{f} \rightarrow \nu_{\sigma}$ (note that the terminology implies that $\phi$ covers the identity map of $M$ ) such that $\phi_{*}\left(\alpha_{f}\right)=\alpha_{g}$. We say $\phi$ induces an equivalence between the normal invariants of $f$ and $g$.

THEOREM 2. Suppose $2 m>3(n+1)$. Then two imbeddings of $M$ in $S^{m}$ are isotopic if and only if they have equivalent normal invariants.

Theorems 1 and 2 represent alternatives to the classification theorems of [5]. Note that the normal bundle plays a more prominent role here; in particular, Theorem 1 gives us information on the possible normal bundles of imbeddings.

The situation is more complicated in the borderline case $2 m$ $=3(n+1)$. For $n=4 k-1, m=6 k$, we obtain a generalization of the main result of $[4]$. Let $(\xi, \alpha)$ be the normal invariants of an imbedding of $M$ in $S^{m} ;$ we will define a cyclic group $Z(\xi, \alpha)$. If $f, g$ are imbeddings of $M$ in $S^{m}$ whose normal invariants are equivalent to $(\xi, \alpha)$ we define a further invariant $L(f, g) \in Z(\xi, \alpha)$.

For the following theorem we must impose an additional restriction upon $M$ :

$\left(^{*}\right)$ If $H$ is a homotopy $n$-sphere which bounds a $\pi$-manifold and the connected sum $M \# H$ is diffeomorphic to $M$, then $H$ is diffeomorphic to $S^{n}$.

THEOREM 3. Suppose $n=4 k-1, m=6 k$.

(a) If $f, g$ are imbeddings of $M$ in $S^{m}$ with equivalent normal invariants, then $f$ and $g$ are isotopic if and only if $L(f, g)=0$.

(b) If $f$ is an imbedding with normal invariants $(\xi, \alpha)$ and $L \in Z(\xi, \alpha)$, then there exists an imbedding $g$ whose normal invariants are equivalent to $(\xi, \alpha)$ such that $L(f, g)=L$.

Thus $L(f, g)$ plays the role of a difference cochain in obstruction theory. One may conjecture on the existence of a higher obstruction theory for imbeddings with equivalent normal invariants in the "nonstable" range of dimensions.

4. Discussion of proofs. We use a nonstable version of the procedures introduced in $[2 ; 9]$ (see [8] for details). To prove Theorem 1 we construct a submanifold $N$ of $S^{m}$, and a map $h: N \rightarrow M$ of degree +1 such that $h^{*} \xi$ is the normal bundle of $N$ in $S^{m}$. By a suitable generalization of the techniques in $[4, \S 3]$, we can perform spherical modifications on the pair $\left(S^{m}, N\right)$, at each stage defining a new map $h$ so that $h^{*} \xi$ is still the normal bundle. We must use the restriction 
on codimension here. Following $[2 ; 8]$ we can eventually make $h$ a homotopy equivalence, if $n=6,14$ or $n \neq 2 \bmod 4$. If $n \equiv 2 \bmod 4$, we must replace $S^{m}$ by the $m$-disk $D^{m}$ and let $N$ be a bounded manifold imbedded in $D^{m}$, with $\partial N$ a homotopy sphere and $h: N \rightarrow M_{0}$ such that $h^{*}\left(\xi \mid M_{0}\right)$ is the normal bundle to $N$ in $D^{m}$. Now we can perform spherical modifications as above to make $h$ a homotopy equivalence. Using the results of $[3 ; 6]$ one can deform a homotopy inverse of $h$ into an imbedding of $M$ (or $M_{0}$ ) into $S^{m}$ (or $D^{m}$ ) with a normal bundle fibre homotopy equivalent to $\xi$ (or $\xi \mid M_{0}$ ). If $n \equiv 2 \bmod 4$, the imbedding of $M_{0}$ into $D^{m}$ can be extended to $M$ into $S^{m}$.

Theorem 2 is approached by a similar combination of the methods of [9] (see [8]) and the techniques of [4]. At one point we need the following result, which follows easily, in this range of dimensions, from the results of [3]. Let $H$ be a homotopy $n$-sphere and $g: M \rightarrow M \# H$ a diffeomorphism homotopic to the standard homeomorphism. Let $f$ be an imbedding of $M$ in $S^{m}, i$ an imbedding of $H$ in $S^{m}$ and $f^{\prime}$ the imbedding (unique up to isotopy) of $M \# H$ in $S^{m}$ induced by $f$ and $i$. Then $f^{\prime} \cdot g$ is isotopic to $f$.

The proof of Theorem 3 proceeds as that of Theorem 2 up to a point. In order to complete the necessary spherical modifications we must consider a linking number invariant (an integer) similar to that defined in [4]. After reducing to a quotient group, $Z(\xi, \alpha)$, we obtain $L(f, g)$ which depends only on $f$ and $g$. Now, the verification of (a) is not unlike the arguments in $[4, \S 3] ;(b)$ is proved by adjoining to $f$ one of the knotted spheres constructed in [4].

5. More general results. Given aclosed $m$-manifold $V$ and $v \in H_{n}(V)$, we may ask whether $v$ can be realized by an imbedding $f$ of $M$. If so, we can define the normal bundle $\nu_{f}$ and $\alpha_{f} \in \pi\left(V, T\left(\nu_{f}\right)\right.$ ) (=homotopy classes of maps $\left.V \rightarrow T\left(\nu_{f}\right)\right)$, using the procedures of [10], such that $\alpha_{f}^{*}\left(u\left(\nu_{f}\right)\right)=$ dual of $v$ (if $\xi$ is a $k$-plane bundle over $M, u(\xi) \in H^{k}(T(\xi)$ ) is the usual generator). Also $f^{*} \tau_{V}=\tau_{M}+\nu_{f}$, where $\tau_{M}$ denotes the tangent bundle of $M$. In particular, if $n=4 k$ and $p_{i}(V)=0$ for $0<i<k$ :

$$
\text { index } M=L_{k}\left(\bar{p}_{1}(\xi), \cdots, \bar{p}_{k-1}(\xi), \bar{p}_{k}(\xi)+\left\langle p_{k}(V), v\right\rangle M\right)
$$

where $\bar{p}_{i}(\xi)$ is the dual Pontryagin class of $\xi, L_{k}$ is the Hirzebruch polynomial (see [7]) and $\xi=\nu_{f}$.

Theorem 4. Let $M, V, v$ be as above and $2 m \geqq 3(n+1)$. Assume $\pi_{i}(V)=0$ for $2 i \leqq n$. Suppose $\xi$ is an $(m-n)$-plane bundle over $M$ satisfying ( $\dagger)$ if $n=4 k$ and there exists $\alpha \in \pi(V, T(\xi))$ such that $\alpha^{*}(u(\xi))$ 
$=d u a l$ of $v$. Then there is an imbedding $f$ of $M$ in $V$, representing $v$, such that $\nu_{f}$ is fiber homotopy equivalent to $\xi$ :

(a) Over $M$ if $n=6,14$ or $n \not \equiv 2 \bmod 4$.

(b) Over $M_{0}$ if $n \equiv 2 \bmod 4$.

The proof is similar to that of Theorem 1. There is also an isotopy theorem.

\section{BIBLIOGRAPHY}

1. M. Atiyah, Thom complexes, Proc. London Math. Soc. (3) 11 (1961), 291-310.

2. W. Browder, Homotopy type of differentiable manifolds, pp. 42-46, Colloquium on Algebraic Topology, Aarhus, 1962.

3. A. Haefliger, Plongements différentiables de variêtés dans variêtés, Comment. Math. Helv. 36 (1961), 47-82. 466.

4. - Knotted (4k-1)-spheres in 6k-space, Ann. of Math. (2) 75 (1962), 452-

5. - Plongements différentiables dans le domaine stable, Comment. Math. Helv. 37 (1962), 155-176.

6. M. Hirsch, On the fibre homotopy type of normal bundles of manifolds, (unpublished).

7. M. Kervaire and J. Milnor, Groups of homotopy spheres. I, Ann. of Math. (2) 77 (1963), 504-537.

8. R. Lashof, Some theorems of Browder and Novikov on homotopy equivalent manifolds with an application, Printed Notes, Univ. of Chicago, Chicago, Illinois.

9. S. P. Novikov, Diffeomorphisms of simply-connected manifolds, Soviet Math. Dokl. 3 (1962), 540-543.

10. R. Thom, Quelques propriêtés globales des variétés différentiables, Comment. Math. Helv. 28 (1954), 17-86.

Massachusetts Institute of Technology 